\title{
SCP-Trust Reasoning Strategy Based on Preference and Its Service Composition Process of Context-Aware Process
}

\author{
Xiaona Xia, Jiguo Yu, Baoxiang Cao \\ School of Computer Science, Qufu Normal University, Rizhao, China \\ Email: xiaxn@sina.com
}

Received May 2014

\begin{abstract}
Before providing services to the user, user preference considerations are the key conditions to achieve the self-adaptive decision-making about service selection and composition process, which is the flexible concerned aspect provided by massive cloud computing environment data. Meanwhile, during the whole services' providing process, achieving the capturing and forming of service aggregation units' topology logic, building the context environment's process-aware of service composition, ensuring the trust and adaptation among service aggregation units, which are the important reasons to express timely requirement preference. This paper designs SCP-Trust Reasoning strategy about the integration of user preference and trust, with process algebra, it is to achieve the context process-aware logic for service composition process, in order to improve the autonomous optimization and evolution of service implementation system.
\end{abstract}

\section{Keywords}

Preferences, Trust Reasoning, Context-Aware Process, Service Composition, Service Aggregation Unit

\section{Introduction}

\subsection{Service and Cloud Technology}

With the rapid development of service and cloud computing, the business organization and topology building based Web services have gotten attention extensively [1]-[4]. The integration of the two technologies make cloud services more rich, there are a large number of service resources in cloud, while it is difficult to select services for timely user requirements according to preferences, how to adaptively make out the countermeasures and topology implementation sequences by effectively analyzing preferences is the achievement goal of autonomous service selection and composition. However, the information interaction between users and service resource pool, some information will often be missed or ignored as noise, which might cause imperfect requirement conditions about service selection and composition, and does not fully reflect the trust and preferences, and even deviate from the real requirement substance, leading to the topological implementation results are not re- 
quired by users, seriously, it causes the lack of information and destruction.

\subsection{Related Work}

Zeng et al. [4] proposed a global optimization method based on integer rules, with the help of context requirement, one optimal implementation path was selected; Ardagna and Pernic [5] concerned the global and local issues about services selection; Yu and Lin [6] used the requirement optimization and result of knapsack problem to achieve composition services selection; Korkmaz and Krunz [7] put forward a kind of heuristic composition services selection options; Driven by architecture implementation goal, Canfora et al. [8] used genetic algorithms to solve services selection process; Fan Xiaoqin et al. [9] designed a kind of discrete particle swarm algorithm to improve services' response capacity about timely requirement.

\section{Preferences Requirement Principle}

Based on the preference right re-determine method of rough sets [10] [11], capturing preference process is built in the platform, and does the grasping and learning timely during the interaction between users and services, that is the pre-conditions about preferences and services.

Definition 1 (Preference Knowledge Supporting System). Four-tuple $p S=(U, A, V, f)$ stands for this system. $U$ is the non-empty finite object set; $A$ is the non-empty finite attribute set, $A=C \cup D, C \cap D=\phi$. $C$ is the condition attribute set, $D$ is decision-making supporting attribute set; $V=\bigcup_{\alpha \in A} V_{\alpha}, V_{\alpha}$ is range of $\alpha$ $(\alpha \in A) ; \quad f: U \times A \rightarrow V$ is a information function, it might give the information domain for every preference attribute.

Definition 2 (Weight Re-measure of Preference attribute): Supposing $X \subset U$ is one subset of attribute, $x_{i} \in U$ is a specific description, after adding specific value $x_{i}$ in $X$, the weight of service selection is improved, the greater of improving degree, $x_{i}$ is more important for $X$ preferences, the important degree of attribute of $x_{i}$ for $X$ is defined as: $\operatorname{pSig}_{x_{i}}\left(x_{i}\right)=1 \frac{\left|\operatorname{pos}_{X x_{i}}(U)\right|}{\left|\operatorname{pos}_{X}(U)\right|}$

If $P$ and $Q$ is the equivalence relationship in $U$, the positive domain of $P$ for $Q$ is denoted as posp $(Q)$, $\operatorname{posp}(Q)=\bigcup_{X \subset U / Q} P X, P$ means that all classified results might be collected in the preference object sets of relationship $Q$ according to $U / P$.

Objective preference weight is described as:

$$
p G_{i}=\frac{p \operatorname{Sig}\left(x_{i}\right)}{\sum_{i=1}^{n} p \operatorname{Sig}_{x_{i}}\left(x_{i}\right)}
$$

Objective weight is basic value, the comprehensive weight will be gotten:

$$
p I(\omega)=\alpha Q(\omega)+(1-\alpha) p G(\omega), \quad 0 \leq \alpha \leq 1
$$

$\alpha$ is experience factor of preference, $0 \leq \alpha \leq 1$ expresses the preference degree for subjective and objective weight in selecting services, the greater $\alpha$, the more important is the ensured requirement branch; the smaller $\alpha$, the implementation of requirement cares for objective weight more. When $\alpha=1$, the selection process only thinks about current requirement goals, does not do the cognitive exhibition; if $\alpha=0$, it is needed to consider objective weight and be aware of the learning and evolution analysis about requirement goals.

\section{Preference-Driven Trust Relationship and Evaluation}

In order to extend and deepen the cognitive range of service customer $S C$, thereby enhancing complex synergy and the accuracy of service scheduling in different platforms, and increasing cross-organizational the effectiveness of service selection and composition.

Assume that service provider of $S P$ about service customer $A$ of $S C$ is $\left[S P_{1}, S P_{2}, \cdots, S P_{m}\right]$, if the number of service customers interacting with $m S P$ is $n$, under the premise of not involving preferences, its interactive evaluation records might be expressed as follows matrix structure: 


$$
S Q_{A, 1 . . m}^{1 . n}=\left[\begin{array}{ccccc}
S Q_{1,1}^{r} & S Q_{1,2}^{r} & S Q_{1,3}^{r} & \ldots & S Q_{1, m}^{r} \\
S Q_{2,1}^{r} & S Q_{2,2}^{r} & S Q_{2,3}^{r} & \ldots & S Q_{2, m}^{r} \\
\ldots & \ldots & \ldots & \ldots & \ldots \\
S Q_{i, 1}^{r} & S Q_{i, 2}^{r} & S Q_{i, 3}^{r} & \ldots & S Q_{i, m}^{r} \\
\ldots & \ldots & \ldots & \ldots & \ldots \\
S Q_{n, 1}^{r} & S Q_{n, 2}^{r} & S Q_{n, 3}^{r} & \ldots & S Q_{n, m}^{r}
\end{array}\right]
$$

Every element in matrix $S Q_{A, B}^{r}$ is the evaluation vector of the different service times, which is equivalent to $S Q_{A, B}^{r}=\left[S Q_{A, B}^{(t 1)}, S Q_{A, B}^{(t 2)}, S Q_{A, B}^{(t 3)}, \cdots, S Q_{A, B}^{(t x)}\right]$, the same time, every element of vector $S Q_{A, B}^{r}$ is also a multidimensional vector, when it is expressed as $S Q_{A, B}^{t j}=\left[S Q_{A, B}^{1,(t j)}, S Q_{A, B}^{2,(t)}, S Q_{A, B}^{3,(t)}, \cdots, S Q_{A, B}^{v,(t j)}\right]$, represents that $S Q_{A, B}^{t j}$ is one $v$-dimensional vector.

In matrix $S Q_{A, 1 . . m}^{1 . n},\left[S Q_{j, 1}^{r}, S Q_{j, 2}^{r}, S Q_{j, 3}^{r}, \cdots, S Q_{j, m}^{r}\right]$ express $Q o S$ of $m$ service provider entities for this set of values might be recognized as $m S P$ service providers in line with the actual target values, it might verify whether other $S C$ service customer reflect QoS of SP service providers, furthermore, it might reduce its trust values:

$$
S Q_{A, B}^{r}=\left[S Q_{A, B}^{(t 1)}, S Q_{A, B}^{(t 2)}, S Q_{A, B}^{(t 3)}, \cdots, S Q_{A, B}^{(t x)}\right]
$$

This formula represents $Q O S$ of $S C$ service entity $A$ for $S P$ service provider $B$. Take any one element from $S Q_{A, 1 . . m}^{1 . n}, S Q_{C, B}^{r}=\left[S Q_{C, B}^{(t 1)}, S Q_{C, B}^{(t 2)}, S Q_{C, B}^{(t 3)}, \cdots, S Q_{C, B}^{(t x)}\right]$, this formula $Q o S$ of $S C$ service customer $C$ for $S P$ service provider $B$, because $S C$ service customer $C$ really completes its own evaluation, so that, it might justify the trust of $S C$ service customer $C$ according to the differences' evaluation of $S C$ service customer for $S P$ service provider $B$. From $S Q_{A, B}^{r}$ and $S Q_{C, B}^{r}$, the evaluation vector of $S C$ service customer $A$ and $S C$ entity $C$ for $S P$ service provider $B$ about tj moment:

$$
\begin{aligned}
S Q_{A, B}^{t j} & =\left[S Q_{A, B}^{1,(t j)}, S Q_{A, B}^{2,(t j)}, S Q_{A, B}^{3,(t j)}, \cdots, S Q_{A, B}^{v,(t)}\right] \\
S Q_{C, B}^{t j} & =\left[S Q_{C, B}^{1,(t j)}, S Q_{C, B}^{2,(t j)}, S Q_{C, B}^{3,(t j)}, \cdots, S Q_{C, B}^{v,(t)}\right]
\end{aligned}
$$

Combining with the preferences analysis of the second part, the evaluation difference based on preferences is derive:

$$
\Delta_{A, C}^{B,(t j)}=\Delta(A, C, B, t j)=p I(\omega) \cdot \sum_{k=1}^{v} \tilde{\omega}_{m}\left|Q_{A, B}^{k, t j}-Q_{C, B}^{k, t j}\right|
$$

Further, the ratio of the difference in value of the trust might be calculated as:

$$
\begin{aligned}
& \varphi_{A, C}^{B,(t j)}=\Delta_{A, C}^{B,(t)} / \sum_{k=1}^{v} \tilde{\omega}_{m} Q_{A, B}^{k, t j} \\
& C_{A, C}^{B,(t j)}=1+\left\lceil\Lambda-\psi_{A, C}^{B,(t j)}\right\rceil \cdot \partial
\end{aligned}
$$

In Formula (6), $\partial$ represents the component of gradient, “ \lceil\rceil ” expresses arithmetic modulo, $\Lambda$ is constant value. When difference is smaller that threshold constant $\Lambda$, it means credible, the smaller the difference value, the higher the trust. When the difference value is greater than $\Lambda$, it means incredible, its trust values is negative. Thus through $S P$ entity $B$, about $S P$ service provider $S C$ entity $C$, the trust evaluation of $S P$ service customer $A$ has the information evaluation vector:

$$
C_{A, C}^{B}=\left[C_{A, C}^{B,(t 1)}, C_{A, C}^{B,(t 2)}, \cdots, C_{A, C}^{B,(t)}\right]
$$

Through the trust level method, if the trust evaluation $C_{A, C}^{B,(t)} \geq 0$, trust density value is 1 , and 0 otherwise. requirement preference is the premise, the trust evaluation $f_{A, C}^{B}$ of other $n S C$ is equivalent to $p I(\omega) \cdot f\left(\alpha_{A, C}^{B}, \beta_{A, C}^{B}, \gamma_{A, C}^{B}\right)$, it is refined as the following matrix structure: 


$$
f_{A, 1 . . m}^{1 . n}=\left[\begin{array}{ccccc}
f_{j, 1}^{1} & f_{j, 1}^{2} & f_{j, 1}^{3} & \ldots & f_{j, 1}^{m} \\
f_{j, 2}^{1} & f_{j, 2}^{2} & f_{j, 2}^{3} & \ldots & f_{j, 2}^{m} \\
\ldots & \ldots & \ldots & \ldots & \ldots \\
f_{j, j}^{1} & f_{j, j}^{2} & f_{j, j}^{3} & \ldots & f_{j, j}^{m} \\
\ldots & \ldots & \ldots & \ldots & \ldots \\
f_{j, m}^{1} & f_{j, m}^{2} & f_{j, m}^{3} & \ldots & f_{j, n}^{n}
\end{array}\right]
$$

\section{SCP-TP (Service Composition Preference-Trust Reasoning)}

SCP-TP is described as the follows: according to goal's preferences, service entity $S A$ might reduces its own trust relationship, which is showed as the interaction relationship between $S A$ and $S C_{0}$, the whole interaction accepts the preference-driven effect of PIG (Platform Implementation Goal). SA is one set of SP entities interacted with $S C$ entities $A, S C_{0}$ is one set of $S C$ entities interacted with $S P$ entities in $S A$ set. Because the preference implementation condition led by goal's changing is the trust logic of $S P$ of $S P_{k}$ in Figure 1, which might gotten by the indirect trust relationship by PIG.

Trust reasoning and process-aware in Figure 1 is described as follows:

1) Preferences of platform goal and real-time user requirement is mapped for PIG (related characterization described in the paper has been assigned), PIG realization is attributed to cluster logic set of $S C$ service customer entity $A$. The serialization process of $A$ is submitted by $S P_{0}$, and does a series of reasoning that $S C_{0}$ cluster set is the starting point, to get the trust value for $S P_{1}$ cluster set.

2) Besides $S C_{0}$ cluster set, $S C$ interacted with $S P_{1}$ cluster also introduces the reasoning and process-aware with $S C_{1}$ cluster. Contrasting $S C_{0}$ cluster and $S C_{1}$ cluster about the difference of $S P_{1}$ cluster, reduces the trust results of $S C_{1}$. In this process, $S P$ cluster entity is viewed as the reference of $S C$ trust evaluation and reduces $S C_{1}$ trust. In this way, trust reasoning and service goal might reduce $S P_{1}$ cluster and $S C_{1}$ cluster.

3) According to the second step of this process, further reasoning, it might get $Q o S$ weight of $S P_{k}$ cluster that its trust coincides with preferences.

$S C_{0}$ driven by $P I G$ has $n$ entities, there are $z$ entities in $S C_{1}, S P_{0}$ has $m$ entities, $S P_{1}$ is with $y$ entities. Preference $Q o S$ evaluation matrix of $S P_{1}$ set related to $P I G$ is described as:

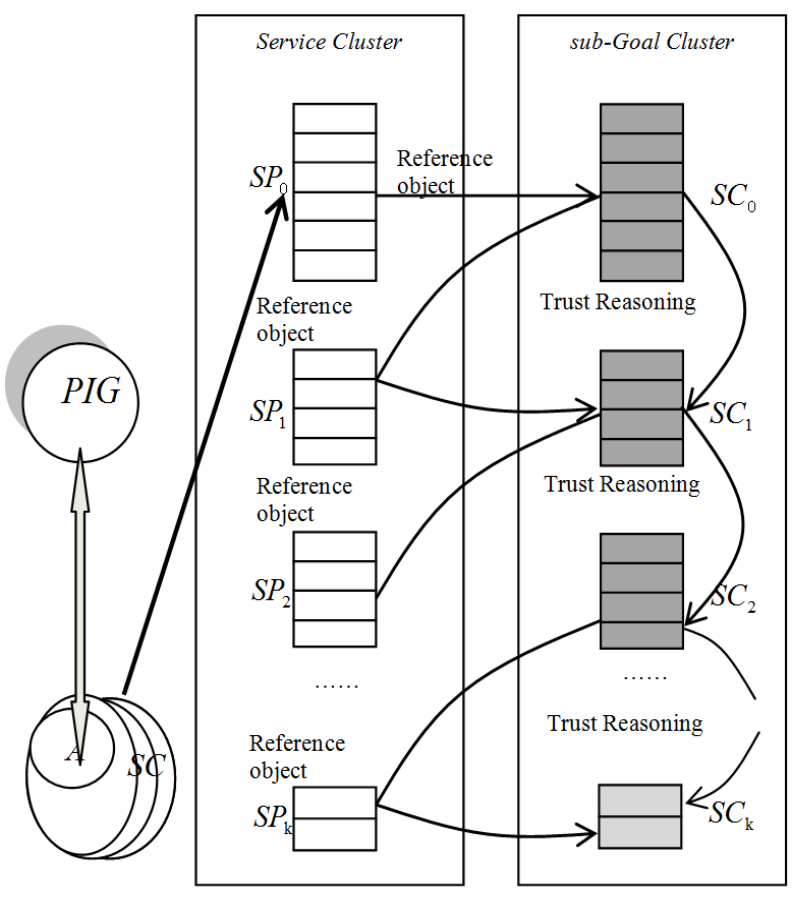

Figure 1. Trust reasoning and process-aware of SCP-TP. 


$$
Q_{0,1 . . n}^{1 . . y}=\left[\begin{array}{ccccc}
Q_{1,1}^{r} & Q_{1,2}^{r} & f_{1,3}^{r} & \ldots & Q_{1, y}^{r} \\
Q_{2,1}^{r} & f_{2,2}^{r} & f_{2,3}^{r} & \ldots & f_{2, y}^{r} \\
\ldots & \ldots & \ldots & \ldots & \ldots \\
Q_{j, 1}^{r} & f_{j, 2}^{r} & f_{j, 3}^{r} & \ldots & f_{j, y}^{r} \\
\ldots & \ldots & \ldots & \ldots & \ldots \\
f_{n, 1}^{r} & f_{n, 2}^{r} & f_{n, 3}^{r} & \ldots & f_{n, y}^{r}
\end{array}\right]
$$

Corresponding to QoS of SP entities adjusted by PIG, it might get the trust evaluation of $n S C$ entities in $S C_{0}$ set according to above reasoning and aware:

$$
T_{0,1 . . n}^{1 . . y}=\left[\begin{array}{cccc}
f\left(\alpha_{1,1}, \beta_{1,1}, \gamma_{1,1}\right) & f\left(\alpha_{1,2}, \beta_{1,2}, \gamma_{1,2}\right) & \ldots & f\left(\alpha_{1, y}, \beta_{1, y}, \gamma_{1, y}\right) \\
f\left(\alpha_{2,1}, \beta_{2,1}, \gamma_{2,1}\right) & f\left(\alpha_{2,2}, \beta_{2,2}, \gamma_{2,2}\right) & \ldots & f\left(\alpha_{2, y}, \beta_{2, y}, \gamma_{2, y}\right) \\
\ldots & \ldots & \ldots & \ldots \\
f\left(\alpha_{i, 1}, \beta_{i, 1}, \gamma_{i, 1}\right) & f\left(\alpha_{i, 2}, \beta_{i, 2}, \gamma_{i, 2}\right) & \ldots & f\left(\alpha_{i, 2}, \beta_{i, 2}, \gamma_{i, y}\right) \\
\ldots & \ldots & \ldots & \ldots \\
f\left(\alpha_{n, 1}, \beta_{n, 1}, \gamma_{n, 1}\right) & f\left(\alpha_{n, 2}, \beta_{n, 2}, \gamma_{n, 2}\right) & \ldots & f\left(\alpha_{n, 2}, \beta_{n, 2}, \gamma_{n, y}\right)
\end{array}\right]
$$

$Q_{0,1 . . n}^{1 . y}$ matrix represents $Q o S$ evaluation of $S C_{0}$ about $S P_{1}, Q_{1,1 . .2}^{1 . y}$ matrix represents $Q o S$ evaluation of $S C_{1}$ about $S P_{1}$. Further might be obtained:

$$
Q_{1,1 . . z}^{1 . . y}=\left[\begin{array}{ccccc}
Q_{1,1}^{r} & Q_{1,2}^{r} & Q_{1,3}^{r} & \ldots & Q_{1, y}^{r} \\
Q_{2,1}^{r} & Q_{2,2}^{r} & Q_{2,3}^{r} & \ldots & Q_{2, y}^{r} \\
\ldots & \ldots & \ldots & \ldots & \ldots \\
Q_{i, 1}^{r} & Q_{i, 2}^{r} & Q_{i, 3}^{r} & \ldots & Q_{i, y}^{r} \\
\ldots & \ldots & \ldots & \ldots & \ldots \\
Q_{z, 1}^{r} & Q_{z, 2}^{r} & Q_{z, 3}^{r} & \ldots & Q_{z, y}^{r}
\end{array}\right]
$$

This process is described as:

Step 1: Calculate the evaluation values of $E$ and $F$ for $S P$ entity $H$, then reduce the trust of $E$ about $F$, the evaluation difference value of $H$ at $t_{i}$ moment is

$$
\eta_{E, F}^{H\left(t_{i}\right)}=\eta\left(E, F, H, t_{i}\right)=\sum_{k=1}^{u} \varpi_{m}\left|Q_{E, H}^{k, t_{i}}-Q_{F, H}^{k, t_{i}}\right|
$$

Step 2: According to the above reasoning and PIG adjustment of $S C$ and $S P$, it might reduce and get the trust about services' participation:

$$
\begin{gathered}
\varphi_{E, F}^{H\left(t_{i}\right)}=P I G \cdot \eta_{E, F}^{H\left(t_{I}\right)} / \sum_{k=1}^{u} \tilde{\varpi}_{m} Q_{E, H}^{k, t_{i}} \\
T_{E, F}^{H\left(t_{I}\right)}=1+\left\lceil\Lambda-\varphi_{E, F}^{H\left(t_{i}\right)}\right] \cdot \sigma
\end{gathered}
$$

The goal trust of service entity $E$ and $F$ during selecting and compositing of $S P$ entity $H$ scheduling by $P I G$ is:

$$
\begin{gathered}
T_{E, F}^{H}=\left[T_{E, F}^{H\left(t_{1}\right)}, T_{E, F}^{H\left(t_{2}\right)}, T_{E, F}^{H\left(t_{3}\right)}, \cdots, T_{E, F}^{H\left(t_{v}\right)}\right] \\
f_{E, F}^{H}(\alpha, \beta, \gamma)=\left[f_{E, F}^{1}(\alpha, \beta, \gamma), f_{E, F}^{2}(\alpha, \beta, \gamma), f_{E, F}^{3}(\alpha, \beta, \gamma), \cdots, f_{E, F}^{y}(\alpha, \beta, \gamma)\right]
\end{gathered}
$$

Further might get every $S C_{1}$ service entity's information evaluation of $S C_{0}$ service entity $E$ by $S P_{1}$ set. Every participation entity's trust evaluation of $S C_{1}$ about $S C$ service entity in $S C_{0}$ is expressed in the left part of Formula (17). $j$ line of Formula (17) expresses the trust evaluation of $j S C_{1}$ entity that service entity $E$ has completed to refer different service provider $S P_{1}$, then it might also get the trust evaluation process of $E$ about every $S C_{1}$ enti- 
ty, it is showed as the right matrix structure in Formula (17).

$$
\left[\begin{array}{cccc}
f_{j, 1}^{1} & f_{j, 2}^{2} & \cdots & f_{j, 1}^{v} \\
f_{j, 2}^{1} & f_{j, 2}^{2} & \cdots & f_{j, 2}^{v} \\
\cdots & \cdots & \cdots & \cdots \\
f_{j, j-1}^{1} & f_{j, j-1}^{2} & \cdots & f_{j, j-1}^{v} \\
\cdots & \cdots & \cdots & \cdots \\
f_{j, 2}^{1} & f_{j, 2}^{2} & \cdots & f_{j, z}^{v}
\end{array}\right] \Rightarrow\left[\begin{array}{c}
f\left(\alpha_{j, 1}^{\text {idir }}, \beta_{j, 1}^{\text {idir }}, \gamma_{j, 1}^{\text {idir }}\right) \\
f\left(\alpha_{j, 2}^{\text {idir }}, \beta_{j, 2}^{\text {idir }}, \gamma_{j, 2}^{\text {idir }}\right) \\
\cdots \\
\cdots\left(\alpha_{j, j-1}^{\text {idir }}, \beta_{j, j-1}^{\text {idir }}, \gamma_{j, j-1}^{\text {idir }}\right) \\
\cdots \\
f\left(\alpha_{j, z}^{\text {idir }}, \beta_{j, z}^{\text {idir }}, \gamma_{j, 2}^{\text {idir }}\right)
\end{array}\right]
$$

Based on the above results, the trust calculation reasoning model driven by preferences between service providers and service customers about PIG is Formula (18):

$$
\left[\begin{array}{cccc}
f\left(\alpha_{1,1}^{\text {idir }}, \beta_{1,1}^{\text {idir }}, \gamma_{1,1}^{\text {idir }}\right), & f\left(\alpha_{1,2}^{\text {idir }}, \beta_{1,2}^{\text {idir }}, \gamma_{1,2}^{\text {idir }}\right), & \cdots, & f\left(\alpha_{1,2}^{\text {idir }}, \beta_{1, z}^{\text {idir }}, \gamma_{1,2}^{\text {idir }}\right) \\
f\left(\alpha_{2,1}^{\text {idir }}, \beta_{2,1}^{\text {idir }}, \gamma_{2,1}^{\text {idir }}\right), & f\left(\alpha_{2,2}^{\text {idir }}, \beta_{2,2}^{\text {idir }}, \gamma_{2,2}^{\text {idir }}\right), & \cdots, & f\left(\alpha_{2, z}^{\text {idir }}, \beta_{2,2}^{\text {idir }}, \gamma_{2,2}^{\text {idir }}\right) \\
\cdots & \cdots & \\
f\left(\alpha_{n, 1}^{\text {idir }}, \beta_{n, 1}^{\text {idir }}, \gamma_{n, 1}^{\text {idir }}\right), & \left(\alpha_{n, 2}^{\text {idir }}, \beta_{n, 2}^{\text {idir }}, \gamma_{n, 2}^{\text {idir }}\right), & \cdots, & \left(\alpha_{n, z}^{\text {idir }}, \beta_{n, z}^{\text {idir }}, \gamma_{n, 2}^{\text {idir }}\right)
\end{array}\right]
$$

The first line of Formula (18) is corresponding to the first column of the right part in Formula (18), that expresses the corresponding trust evaluation of some service customer entity $S C$ for every entity in $S C_{1}$ set. According to the above logical derivation process, $S C_{0}$ is the starting point, $S P_{1}$ deduces trust value of $S C_{0}$ for $S C_{1}$, then, $S C_{0}$ is as the starting point, $S P_{2}$ deduces trust value for $S C_{2}$, it might get the trust about $S C_{k}$ in Figure 1.

Through the above derivation process, we might obtain the QoS acquisition approach of QoS that there $S P$ interacted with $S C$ indirectly, and $Q o S$ evaluation model that direct interaction trust is precondition and user preferences are the important factors adjusted by PIG.

The trust and process-aware in Figure 1, according the above analysis and argumentation, temporary indirect trust about service selection and composition might get the transformation and indirect transition in Figure 2. This process might be described as: first, $S C$ entity $S$ might get the trust relationship of $S C_{0}$. Secondly, according to the forward reasoning, it might get $S P_{0}$ entity $D$ adjusted by PIG. Based on service composition and PIG, transition relationship of trust in Figure 2 achieves other indirect transition routing of trust.

\section{Service Composition Process Based on Process-Aware}

Process topology and implementation semantic logic of CAPT, this part gives CCML description.

$<$ CCML: Composition>

$<$ CCML: sequence $>$

$<!--$ read channel-->

$<$---get PIG implementation decision-making-->

$<$ CCML: Capture the activities about PIG, which are managed in some way $>$

$<$ !--read service transition channel based on the goal of some activity-->

<CCML: readChannel Channel="PIG_channelName" Service="PIG_serviceName” action="PIG_actionName" port="portName” $>$

$<$ !--implement one service activity-->

$<$ !--process-aware logic of PIG reads the context requirement and implement from corresponding service register channel. -->

$<!--$ read service register channel-->

$<$ CCML: readReg reg="PIG_registerName" Service="PIG_ServcieName" action="PIG_actionName” port= "portName">

$<$ CCML: execute action="PIG_actionName” Service="PIG_serviceName”>

$<$ !--write channel-->

<CCML: writeChannel Channel="PIG_channelName" Service="PIG_serviceName” action="PIG_actionName” port="portName" $>$ 

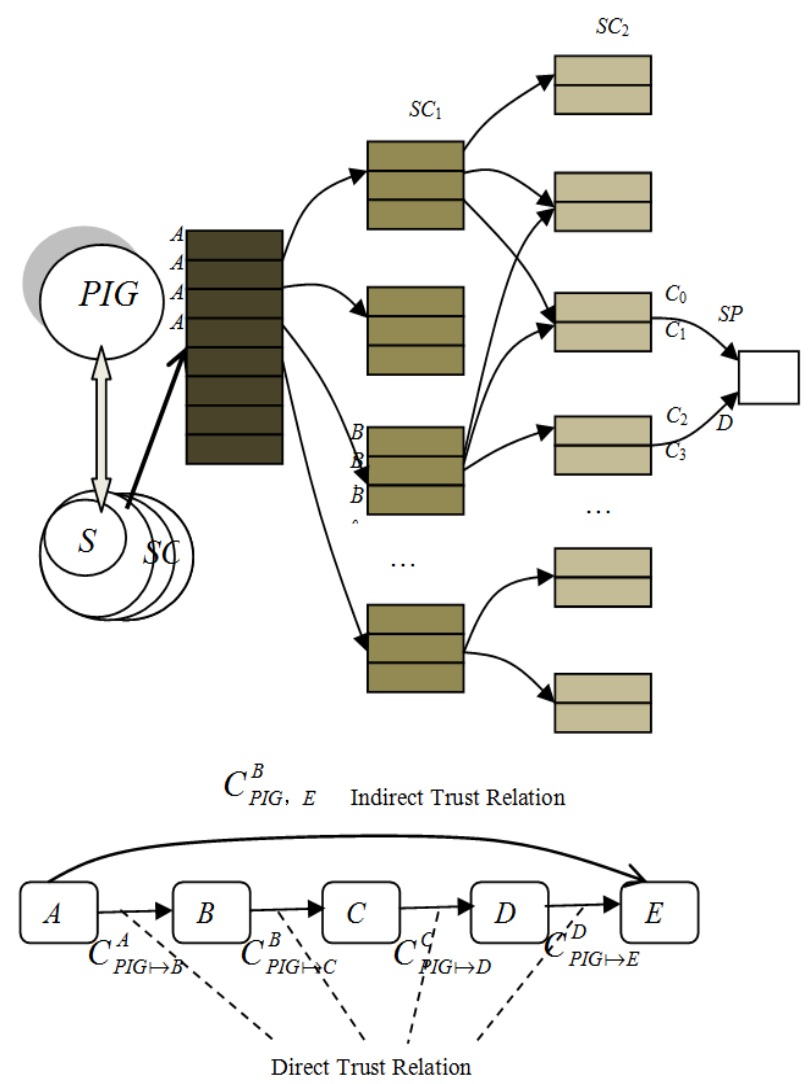

Figure 2. Transformation and transition of trust.

$<$ !--write service register channel-->

<CCML: writeReg reg="PIG_registerName" Service="PIG_ServcieName" action="PIG_actionName" port= "portName" $>$

<CCML: execute action="PIG_actionName" Service="PIG_serviceName">

$</$ CCML: sequence>

$</$ CCML: composition>

\section{Conclusions}

Thinking about preferences of service selection and composition, this paper analyzes and argues the trust reasoning strategies and implementation measures of service flow platform. For goal preferences and topology effectiveness, this paper designs and achieves process-aware approach that PIG is the main adjustment mechanism, then gives the algorithm and adjustment platform logic for actual implementation case.

In processing of problems, timely goal preferences of goal and trust reasoning of flow are the key problem in service computing and cloud computing, PIG fully considers goal preferences and topology trust, and reduces SCP-strategy logic, and achieves the context process-aware logic and implementation process. Through actual case's R\&D and optimal project of platform, it improves the autonomous optimization and evolution capacity of service flow.

\section{Acknowledgements}

This paper is supported by National Natural Science Foundation of China (61373027, 11101243), Natural Science Foundation of Shandong Province of China (ZR2012FQ011, ZR2012FM023, ZR2009GM009), the Key Science-Technology Development Project of Shandong Province of China (No. 2009GG10001014, 2012GGX10123) and Promotional Foundation (2005BS01016) for Middle-aged or Young Scientists of Shandong Province, SRI of SPED (J12LN06, J07WH05), DRF and UF (3XJ200903, XJ0609) of QFNU. 


\section{References}

[1] Zhang, J.-L. and Fan, Y.-S. (2010) Service-Oriented Enterprise and Business Ecosystem. Computer Integrated Manufacturing Systems, 16, 1751-1759.

[2] Xu, L.-D., Liu, H.-M., Wang, S., et al. (2009) Modeling and Analysis Techniques for Cross-Organizational Workflow Systems. Systems Research and Behavioral Science, 26, 367-389. http://dx.doi.org/10.1002/sres.978

[3] Armbrust, M., Fox, A., Griffith, R., et al. (2010) A View of Cloud Computing. Communications of the ACM, 53, 5058. http://dx.doi.org/10.1145/1721654.1721672

[4] Zeng, L.Z., Benatallah, B., Hgu, A.H.H., et al. (2004) QoS-Aware Middleware for Web Services Composition. IEEE Trans on Software Engineering, 30, 311-327. http://dx.doi.org/10.1109/TSE.2004.11

[5] Ardagna, D. and Pernici, B. (2005) Global and Local QoS Guarantee in Web Service Selection. Proceedings of Business Process Management Workshops, Springer, Berlin, 32-46.

[6] Yu, T. and Lin, K. (2005) Service Selection Algorithms for Composing Complex Services with Multiple Qos Constraints. Proceedings of the 3rd International Conference on Service Oriented Computing, Springer, Berlin, 130-143.

[7] Kormza, T. and Krunz, M. (2001) Multi-Constrained Optimal Path Selection. Proceedings of the 20th Joint Conference of IEEE Computer and Communications, Piscataway, 834-843.

[8] Canfora, G., Penta, M.D., Esposito, R., et al. (2005) An Approach for QoS-Ware Service Composition Based on Genetic Algorithms. Proceedings of the 2005 Conference on Genetic and Evolutionary Computation, ACM, New York, 1069-1075.

[9] Fan, X.Q., Jiang, C.J., Fang, X.W., et al. (2010) Dynamic Web Service Selection Based on Discrete Particle Swarm Optimization. Journal of Computer Research and Development, 47, 147-156.

[10] Beynon, M. (2001) Reduces within the Variable Precision Rough Sets Model: A Further Investigation. European Journal of Operational Research, 134, 592-605. http://dx.doi.org/10.1016/S0377-2217(00)00280-0

[11] Pomykala, J. (1988) The Stone Algebra of Rough Sets. Bulletin of the Polish Academy of Sciences, 36, 495-507. 